\title{
Electrostatic immobilisation of copper(I) and copper(II) bis(oxazolinyl)pyridine catalysts on silica: application to the synthesis of propargylamines via direct addition of terminal alkynes to imines
}

\author{
Chiara McDonagh, ${ }^{a}$ Peter O'Conghaile, ${ }^{a}$ Robertus J. M. Klein Gebbink ${ }^{b}$ \\ and Patrick O'Leary ${ }^{\mathrm{a}, *}$ \\ ${ }^{a}$ National University of Ireland, Galway, Department of Chemistry, Galway, Ireland \\ ${ }^{\mathrm{b}}$ Utrecht University, Organic Chemistry and Catalysis, Faculty of Science, Padualaan 8, 3584 CH Utrecht, The Netherlands
}

Received 22 February 2007; revised 11 April 2007; accepted 18 April 2007

Available online 22 April 2007

\begin{abstract}
Copper(I) and copper(II) complexes of two bis(oxazolinyl)pyridines were immobilized on silica via electrostatic interactions. The catalytic activity of the immobilized catalysts in the direct addition of terminal alkynes to imines leading to propargylamines was investigated under a variety of reaction conditions. The performance of the immobilized catalysts compares very well with their homogeneous equivalents. When used in toluene, the catalysts could be recycled a number of times and maintained activity. This study is the first such report of the immobilization on silica in this manner of any bis(oxazolinyl)pyridine (pybox) complex. (C) 2007 Elsevier Ltd. All rights reserved.
\end{abstract}

\section{Introduction}

As enantioselective catalysis becomes more developed, preparation of many of the ligands and catalytic species involves multistep syntheses of increasing complexity starting from chiral materials such as amino acids. This in turn results in an increase in the cost of such catalysts. Many of these catalytic species offer significant improvements on their simpler predecessors in terms of yield, enantioselectivity or reactions which can be accessed. Some reactions can be carried out using a very low catalyst loading and as such, the expense of the catalyst becomes less important. However, as is the case in the three component coupling to produce propargylamines described here, there remain reactions which require a significant loading of catalyst to progress satisfactorily. ${ }^{1}$

We have recently described a new technique for the electrostatic immobilisation of copper(II) bisoxazolines on silica. $^{2}$ Catalytic species immobilised by this method

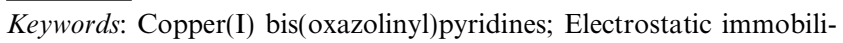
sation; Propargylamine; Silica.

* Corresponding author. Tel.: +35391 492476; fax: +353 91 525700; e-mail: patrick.oleary@nuigalway.ie were used successfully in Diels-Alder cycloadditions. In these reactions the immobilised catalysts were found to give similar yields and enantioselectivities to the homogeneous catalyst. In certain Diels-Alder reactions, the opposite enantiomer was produced using the immobilised catalyst compared to the homogeneous catalyst. The catalyst could be recycled successfully three times.

In seeking to determine the general applicability of our electrostatic immobilisation procedure to such catalysts and their use we decided to study the three component coupling of an amine, an aldehyde and an acetylene which is an excellent method of making propargylamines (Scheme 1). ${ }^{1,3,4}$ Propargylamines are useful building blocks in organic chemistry and have found use in synthesis due to the fact that elements of their structure are found in natural products ${ }^{5}$ and compounds of pharmaceutical interest. ${ }^{6}$ They have been used in the synthesis

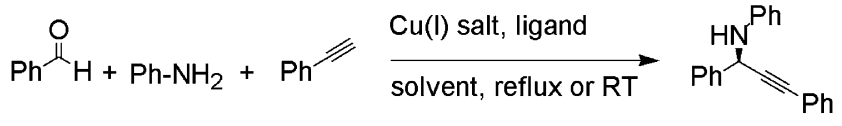

Scheme 1. Three component coupling for the synthesis of propargylamines. 
of pyrrolidines, ${ }^{7}$ oxazoles $^{8}$ and dynemicins. ${ }^{9}$ The obvious advantage of multi-component reactions is the synthesis of a complex molecule from simple starting materials with complete atom economy. A wide variety of compounds are easily accessible given the ease with which the starting materials can be varied. Enantioselective catalysts for this reaction have been developed largely since $2002^{10}$ and avoid the traditional methods for the synthesis of propargylamines which involved stoichiometric amounts of organometallic reagents reacting with an imine. ${ }^{1}$ Some catalytic enantioselective processes have been reported for this reaction but they lack generality and use a large catalyst loading. ${ }^{11,3,4}$ We felt that recyclable catalysts applicable to this reaction would be of use.

Copper(I) complexes of bis(oxazolinyl)pyridines (pybox) are catalytically active for this reaction. Singh has reported a large number of individual reactions for which 5 or $10 \mathrm{~mol} \%$ copper(I) pybox hexafluorophosphates can be used resulting in the isolation of products in high yields and often exceptional enantioselectivity. ${ }^{3}$ Similarly, Li has studied copper(I) pybox trifluoromethanesulfonates and found that they also catalysed the reaction giving high yields and enantiomeric excesses of over $90 \%$ in many cases. ${ }^{11} \mathrm{We}$ are aware of one covalently bound copper catalyst which has been used in the enantioselective catalysis of this reaction. ${ }^{12}$ The necessity to alter the catalyst structure to covalently immobilise it limits any direct comparison which can be made with the homogeneous catalyst. The covalent immobilisation process is often a very arduous process as it requires the synthesis of a special ligand.

We envisaged electrostatic immobilisation of copper(I) and copper(II) pybox trifluoromethane sulfonates on silica which would be the first example of immobilisation of a pybox complex in this way, and also the first example of the immobilisation of such copper(I) complexes and would provide a recyclable catalyst for the synthesis of propargylamines. This would broaden the applicability of this very simple immobilisation technique, the primary advantages of which are the absence of any complexity in the immobilisation process, the widespread availability of silica (chromatographic grade) and the lack of any need to alter the catalyst or ligand to facilitate the immobilisation (Fig. 1).

The phenyl-substituted copper(I) pybox catalyst was first generated in dichloromethane from the commercially available ligand and copper(I) triflate, and this solution was added to silica (silica gel $40-63 \mu \mathrm{m}$, chro- matographic grade) which had been dried under vacuum for $2 \mathrm{~h}$ at $70{ }^{\circ} \mathrm{C} .{ }^{13}$ The heterogeneous mixture was stirred until the colour disappeared from the solvent and the silica became coloured $(\sim 1 \mathrm{~min})$. The organic layer was decanted and the solid washed with two aliqouts of the solvent to be used in the catalytic reaction. Typically, theoretical loadings of $2.8 \%$ copper were used in this study.

The electrostatically immobilised $\mathrm{Cu}(\mathrm{I})$ (phenylpybox) triflate was tested in the reaction of benzaldehyde, aniline and phenylacetylene. Initially, the amine and the aldehyde were combined, without solvent, and heated to $60^{\circ} \mathrm{C}$ for $2 \mathrm{~h}$ and the imine formed was added to the catalyst along with phenylacetylene and toluene. The reaction mixture was then heated to reflux and stirred for $20 \mathrm{~h}$ at which stage the crude reaction mixture could be removed from the catalyst by decanting off the liquid and washing the solid with toluene $(2 \times 5 \mathrm{ml})$. The solid catalyst was now ready to be reused and the product was isolated from the solution by removing the solvent at reduced pressure. The results are shown in Table 1.

The homogeneous $\mathrm{Cu}(\mathrm{I})$ (phenylpybox)triflate catalyst gave $100 \%$ conversion and $51 \%$ ee when the reaction was conducted overnight in refluxing toluene. The $\mathrm{Cu}(\mathrm{I})$ (phenylpybox)triflate catalyst electrostatically immobilised on silica gave very similar results in terms of percentage conversion. The enantioselectivity of the reaction, though reduced in the third use of the catalyst was similar to that of the homogeneous catalyst. This is

Table 1. Immobilised $\mathrm{Cu}(\mathrm{I})$ (phenylpybox) catalysis of propargylamine synthesis

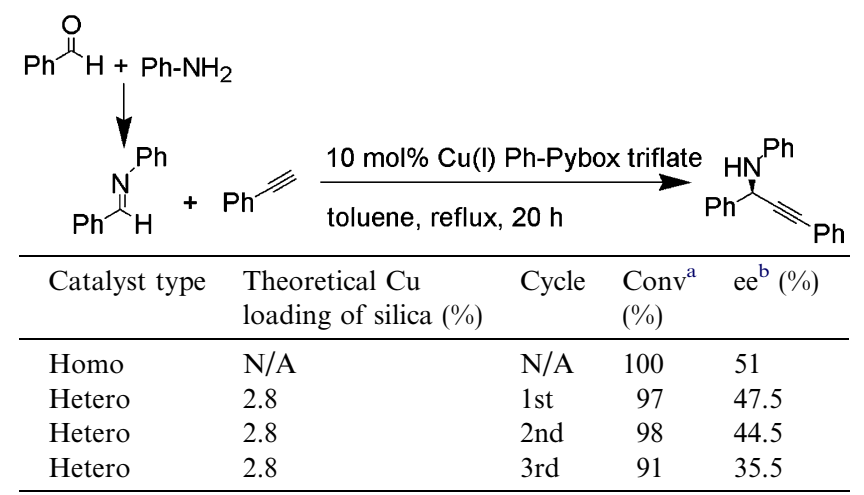

${ }^{\mathrm{a}}$ With respect to the aldehyde determined by ${ }^{1} \mathrm{H}$ NMR analysis.

${ }^{\mathrm{b}}$ Determined by HPLC (Daicel Chiralcel OD, $\left.254 \mathrm{~nm}\right)$, hexane $(0.1 \%$ diethylamine):isopropyl alcohol, 90:10.
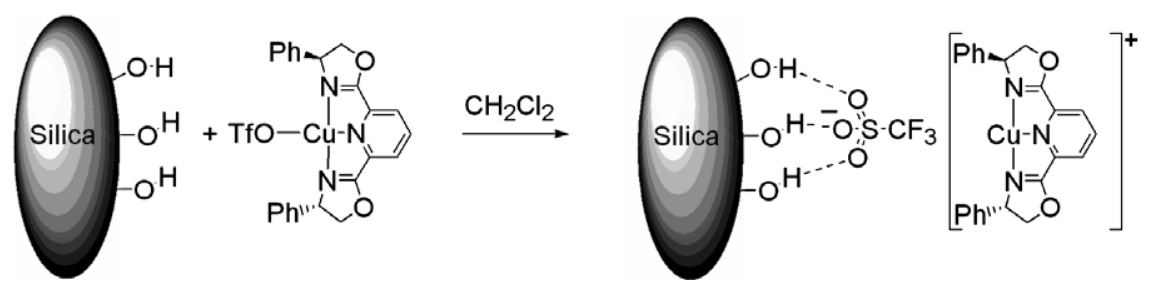

Figure 1. Schematic representation of the immobilisation of a copper(I) pybox catalyst on silica. 
a significant result as it represents a number of new achievements. This is the first reported immobilisation of a copper(I) species in this manner. It is also the first reported immobilisation of a pybox ligand by this method. In addition, it is one of very few enantioselective methods for the catalysis of this reaction using an electrostatically immobilised reusable catalyst. When the immobilised catalyst was refluxed in toluene and the toluene removed and used as solvent for the reaction (in the absence of any further catalyst) no propargylamine was detected indicating that catalysis is generally occurring on the surface and there was minimal catalyst leaching.

We sought to develop this method by varying the amount of catalyst used, the oxidation state of the metal, the substitution on the pybox ligand and the starting aldehyde. The results obtained when the reaction was conducted using $\mathrm{Cu}(\mathrm{I})$ (isopropylpybox) triflate and its silica immobilised equivalent are shown in Table 2. The results using the isopropyl-substituted copper(I) pybox complex were disappointing for both the homogeneous catalyst and its immobilised equivalent. The supported catalyst displayed variable and generally disappointing enantioselectivity but the activity was enhanced as conversions in excess of $90 \%$ were observed in all three runs. Our previous study of $\mathrm{Cu}$ (II) BOX catalysis of Diels-Alder reactions had found that though bis(oxazoline) BOX complexes bearing a phenyl group were stable on immobilisation over multiple uses, those bearing a ${ }^{t}$ butyl group, decomposed during the first use of the immobilised catalyst. In this case the alkyl-substituted pybox ligand survived the immobilisation and several uses.

A study was conducted to see how the $\mathrm{Cu}$ (II) pybox catalysts would perform on immobilisation (Table 3). When the propargylamine synthesis was conducted in dichloromethane at reflux using homogeneous $\mathrm{Cu}$ (II)(phenylpybox), the conversion was very poor but the enantioselectivity was good. When the catalyst was electrostatically immobilised on silica, the performance of the catalyst improved. The percentage conversion increased by $20 \%$ and the enantioselectivity was maintained. On reuse, the immobilised catalyst was less active, and nearly identical to the homogeneous reaction in terms of conversion and enantioselectivity. A similar study of the immobilised catalyst activity in toluene at reflux found that the reaction went to completion and though there was a drop in enantioselectivity in the first

Table 2. Immobilised $\mathrm{Cu}(\mathrm{I})$ (isopropylpybox) catalysis of propargylamine synthesis at reflux in toluene

\begin{tabular}{lllll}
\hline Catalyst type & $\begin{array}{l}\text { Theoretical Cu } \\
\text { loading of silica (\%) }\end{array}$ & Cycle & $\begin{array}{l}\text { Conv } \\
(\%)^{\mathrm{a}}\end{array}$ & $\mathrm{ee}^{\mathrm{b}}(\%)$ \\
\hline Homo & N/A & N/A & 31 & 33 \\
Hetero & 2.8 & $1 \mathrm{st}$ & 98 & 25 \\
Hetero & 2.8 & 2nd & 97 & 17 \\
Hetero & 2.8 & $3 \mathrm{rd}$ & 95 & 30.5 \\
\hline
\end{tabular}

${ }^{a}$ With respect to the aldehyde determined by ${ }^{1} \mathrm{H}$ NMR analysis.

${ }^{\mathrm{b}}$ Determined by HPLC (Daicel Chiralcel OD, $254 \mathrm{~nm}$, hexane $(0.1 \%$ diethylamine):isopropyl alcohol, 90:10.
Table 3. Immobilised $\mathrm{Cu}(\mathrm{II})($ phenylpybox) catalysis of propargylamine synthesis

\begin{tabular}{llllrl}
\hline mol $(\%) \mathrm{Cu}(\mathrm{II})$ & $\begin{array}{l}\text { Catalyst } \\
\text { type }\end{array}$ & Cycle & Solvent & $\begin{array}{l}\mathrm{Conv}^{\mathrm{a}} \\
(\%)\end{array}$ & $\mathrm{ee}^{\mathrm{b}}(\%)$ \\
\hline 5 & Homo & N/A & DCM & 32 & 83.5 \\
5 & Hetero & 1st & DCM & 52 & 83.5 \\
5 & Hetero & 2nd & DCM & 29 & 81.5 \\
5 & Homo & N/A & Toluene & 82 & 62 \\
5 & Hetero & 1st & Toluene & 100 & 56 \\
5 & Hetero & 2nd & Toluene & 100 & 68 \\
10 & Homo & N/A & DCM & 98 & 85 \\
10 & Hetero & 1st & DCM & 99 & 80 \\
10 & Hetero & 2nd & DCM & 76 & 79 \\
10 & Hetero & 3rd & DCM & 52 & 81.5 \\
\hline
\end{tabular}

${ }^{\mathrm{a}}$ With respect to the aldehyde determined by ${ }^{1} \mathrm{H}$ NMR analysis.

${ }^{\mathrm{b}}$ Determined by HPLC (Daicel Chiralcel OD, $254 \mathrm{~nm}$, hexane $(0.1 \%$ diethylamine):isopropyl alcohol, 90:10.

${ }^{\mathrm{c}}$ This reaction was conducted at $40^{\circ} \mathrm{C}$ over $48 \mathrm{~h}$.

run it was improved on the second use of the catalyst to give a similar result to the homogeneous catalyst. This result in itself is surprising given the homogeneous reaction was conducted at $40^{\circ} \mathrm{C}$ and the heterogeneous reaction was conducted at reflux.

Previous reports of the synthesis of propargylamines using homogeneous copper catalysts have used $10 \mathrm{~mol} \%$ of copper so we also investigated the immobilised catalysts performance under these conditions (the loading of copper on the silica was maintained at the same level for this study). The runs using the homogeneous catalyst and the heterogeneous catalyst on first use both gave very high conversions and enantioselectivities of $85 \%$ and $80 \%$ ee, respectively. Though the conversion dropped on the second and third use of the immobilised catalyst, the enantioselectivity was maintained. It is likely that a slightly longer reaction time in the second and third run would allow for high conversions to be achieved in these cases also.

The decrease in activity of the immobilised catalyst on reuse in dichloromethane is thought to be due to copper leaching. Copper was detected by AAS in the product of the reaction corresponding to $<5 \%$ leaching. Reactions in which the solid catalyst was removed at mid reaction did not react further on continued refluxing. This indicates that the copper which does leach is not catalytically active. A similar study with both copper(I) and copper(II) catalysts reacting in toluene showed minimal leaching $(<1 \%)$. Any copper which did leach was found to be catalytically inactive as above. These results account for the catalysts activity being maintained on reuse in toluene but diminishing activity on reuse in dichloromethane.

Finally, to confirm that these results were not just specific to the synthesis of the unsubstituted triphenyl propargylamine the same reaction but using 4-bromobenzaldehyde was studied (Table 4). The heterogeneous catalyst was used for two cycles over the course of which the catalyst remained active even though only $5 \mathrm{~mol} \%$ was used. Most encouragingly, the enantiomeric excess of the product was found to be as good with the 
Table 4. Immobilised $\mathrm{Cu}(\mathrm{I})$ (phenylpybox) catalysis of the synthesis of the bromosubstituted triphenyl propargylamine from 4-bromobenzaldehyde

\begin{tabular}{llll}
\hline Catalyst type & Cycle $^{\mathrm{a}}$ & Conv $^{\mathrm{b}}(\%)$ & $\mathrm{ee}^{\mathrm{c}}(\%)$ \\
\hline $\mathrm{Cu}(\mathrm{I})$ bromide & N/A & 100 & N/A \\
Homogeneous & N/A & 94 & 51 \\
Heterogeneous & 1st & 87 & 53.5 \\
Heterogeneous & 2nd & 71.3 & 66 \\
\hline
\end{tabular}

${ }^{\text {a }}$ Reactions were conducted in toluene at reflux for $20 \mathrm{~h}$.

${ }^{\mathrm{b}}$ With respect to the aldehyde determined by ${ }^{1} \mathrm{H}$ NMR analysis.

${ }^{\mathrm{c}}$ Determined by HPLC (Daicel Chiralcel OD, $\left.254 \mathrm{~nm}\right)$, hexane $(0.1 \%$ diethylamine):isopropyl alcohol, 90:10.

heterogeneous catalyst as with the homogeneous equivalent. Indeed, increased enantiomeric excess was observed on the second cycle with the heterogeneous catalyst which was also superior to the enantioselectivity with the homogeneous catalyst.

In conclusion, the electrostatically immobilised bis(oxazolinyl)pyridine catalysts prepared in this study are the first to be immobilised on silica by this method. This report also contains the first example of a copper(I) based catalyst immobilised by this method. The immobilised catalysts were used in the enantioselective synthesis of propargylamines. High conversions were obtained using the copper(I) catalyst and copper(II) catalysts over a number of cycles. Enantioselectivities were in line with those obtained in the corresponding homogeneous reaction. In toluene, the catalyst activity was maintained over several cycles. In dichloromethane, excellent enantioselectivities were obtained over three cycles but some deactivation due to leaching was observed. This study demonstrates the feasibility of using such electrostatic immobilisation to allow reuse of more specialist (non commercially available) catalysts.

\section{Experimental; heterogeneous catalysis: representative procedure}

A mixture of benzaldehyde ( $50 \mathrm{mg}, 4.72 \times 10^{-4} \mathrm{~mol}$ ) and aniline $\left(53 \mathrm{mg}, 5.70 \times 10^{-4} \mathrm{~mol}\right)$ was heated at $60^{\circ} \mathrm{C}$ for $2 \mathrm{~h}$. Pybox ligand $(5 \mathrm{~mol} \%)$ and copper(I) triflate $(5 \mathrm{~mol} \%)$ were dissolved in dry dichloromethane $(1 \mathrm{ml})$ in a dry Schlenk tube under nitrogen and stirred for $1 \mathrm{~h}$ at $\mathrm{rt}$. The catalyst complex solution was filtered and the filtrate was added to silica gel $(50 \mathrm{mg}, 40$ $63 \mu \mathrm{m}$ pre dried for $1 \mathrm{~h}$ under vacuum at $70^{\circ} \mathrm{C}$ ) in a dry Schlenk tube under nitrogen. The mixture was stirred until the colour had disappeared from the solution ( $\sim 1 \mathrm{~min})$. The silica gel, now coloured, was allowed to settle and washed with dry dichloromethane $(2 \times 5 \mathrm{ml})$ then left under the appropriate dry reaction solvent $(1 \mathrm{ml})$ and nitrogen. The imine mixture was added to the solvent containing the immobilised catalyst complex under nitrogen along with a dry solvent rinse $(0.5 \mathrm{ml})$, followed by phenylacetylene $\left(74 \mathrm{mg}, 7.29 \times 10^{-4} \mathrm{~mol}\right)$. The reaction mixture was stirred at reflux overnight, then allowed to cool to room temperature and the silica gel catalyst was allowed to settle. The solvent layer was removed with a pasteur pipette and filtered through Celite, the remaining solid was then washed with dry solvent $(2 \times 5 \mathrm{ml})$ and the washings filtered through Celite. The silica gel catalyst was left under dry solvent $(1 \mathrm{ml})$ and nitrogen for subsequent catalytic runs. The combined extracts were evaporated under reduced pressure to give a crude product as a yellow oil, which was a mixture of starting materials and product. The percentage conversion was determined by ${ }^{1} \mathrm{H}$ NMR spectral analysis. The product enantiomeric excess was determined by HPLC (Daicel Chiralcel OD, $254 \mathrm{~nm}$ ), hexane $(0.1 \%$ diethylamine):isopropyl alcohol, $90: 10$ at a flow rate of $0.5 \mathrm{ml} / \mathrm{min}$. The major enantiomer was detected at a retention time of $13.3 \mathrm{~min}$ and the minor enantiomer at $16.4 \mathrm{~min}$. The absolute configuration of the product was assigned by analogy to the work of Benaglia. ${ }^{4}$

\section{Acknowledgements}

The authors gratefully acknowledge NUI-Galway for a fellowship (C.M.D.) and Professor M. Hynes for assistance with AAS. This publication has emanated from research conducted with the financial support of Science Foundation Ireland (RFP).

\section{References and notes}

1. Zani, L.; Bolm, C. Chem. Commun. 2006, 4263-4275.

2. O'Leary, P.; Krosveld, N. P.; De Jong, K. P.; van Koten, G.; Klein Gebbink, R. J. M. Tetrahedron Lett. 2004, 45, 3177-3180.

3. Bisai, A.; Singh, V. K. Org. Lett. 2006, 8, 2405-2408.

4. Colombo, F.; Benaglia, M.; Orlandi, S.; Usuelli, F.; Celentano, G. J. Org. Chem. 2006, 71, 2064-2070.

5. Jiang, B.; Xu, M. Angew. Chem., Int. Ed. 2004, 43, 216218.

6. Shibasaki, M.; Ishida, Y.; Iwasaki, G.; Timori, T. J. Org. Chem. 1987, 52, 3488-3489.

7. Havey, D. F.; Sigano, D. M. J. Org. Chem. 1996, 61, 2268-2272.

8. Arcadi, A.; Cacchi, S.; Cascia, L.; Fabrizi, G.; Marinelli, F. Org. Lett. 2001, 3, 2501-2504.

9. Yoon, T.; Shair, M. D.; Danishefsky, S. J.; Schulte, G. K. J. Org. Chem. 1994, 59, 3752-3754.

10. Wei, C.; Li, C. J. J. Am. Chem. Soc. 2002, 124, 5638-5639.

11. Wei, C.; Mague, J. T.; Li, C.-J. Proc. Nat. Acad. Sci. 2004, 101, 5749-5754.

12. Weissberg, A.; Halak, B.; Portnoy, M. J. Org. Chem. 2005 , $70,4556-4559$.

13. Our previous work ${ }^{2}$ involved a Diels-Alder reaction which Evans et al. ${ }^{14}$ have reported fails if water is present on the copper reaction centre. The method of pretreatment of the silica reported here was found to be sufficient to prevent catalyst deactivation indicating that at least free $\mathrm{H}_{2} \mathrm{O}$ was removed.

14. Evans, D. A.; Johnson, J. S.; Olhava, E. J. J. Am. Chem. Soc. 2000, 122, 1635-1649. 